\title{
An investigation to compare the effectiveness of packet loss based on proposed methods in literature
}

\author{
A. A. Rahman*, K. A. Noordin and H. M. Farhad \\ Department of Electrical Engineering, Faculty of Engineering, University of Malaya, 50603 Kuala Lumpur, Malaysia. \\ Accepted 27 February, 2013

\begin{abstract}
Optical flow routing is an optical networks that technique used to ensure that the packet is always in the sequence order. This research is intended to ensure that the network traffic is enhanced of the quality of service (QoS). An analytical method is used in order to show its effectiveness of flow routing. There are three cases that have been investigated in this research investigation namely, packet discards (PD), flow discards (FD) and early flow discards (EFD). The performance metric through put indicated $20 \%$ improvement for early flow discards (EFD) compared to conventional PD by using optical flow routing.
\end{abstract}

Key words: Optical flow routing, packet discard, flow discard and early flow discard.

\section{INTRODUCTION}

A vague introduction is made on packet loss in optical packet-switching networks

In this study, heterogeneous network services and applications as well as the increasing bandwidth demand the future transport network is confronted with the requirements for flexible and dynamic service provisioning, high throughput and assurance of quality of service (QoS). To deal with the challenges, photonic packet switching aims to realize a fast switch of the traffic in the optical domain in a packet-by-packet manner. The fine granularity in the switching is flexible for the support of dynamic service requirements and allows for an efficient resource utilization by taking advantage of the statistical multiplexing gain. In last years, relatively large advancements have been made in the technologies for the fast configurable optical switch fabric. The switching control unit (SCU), however, will keep relying on electronic technologies in the foreseeable future due to the unsolved technological problems in e.g., optical buffering and optical signal processing. Optical packet switching (OPS) and optical burst switching (OBS) are two representative network architectures for the photonic packet switching (Guoqiang Hu, Optical "Quality of Service Guarantee in the Edge Node of Optical Packet/Burst Switched Networks with Traffic Assembly", 2009).

Buffer is required in a router to move packets of data in order to avoid collisions between packet and heading to same destination at the same time.

Data's traffic has been increased rapidly throughout the world, triggered by the recent spread in TCP/IP deployment (Thomas, 1996). Optical packet switching networks (OPSNs) have been extensively studied to support data traffic during the last few years, to achieve high throughput, low packet-lost probability (PLP), low delay and quality of services (QoS) (Guillemot, 1998; Carena, 1998). Using packet switching that is treated as a single entity to be routed and switched. As of the statistical sharing of the bandwidth among packets switching is more flexible and efficient compare to the circuit switching.

Packet lost is happening in optical packet-switching routers (OPSRs) because of the potential packet conflicts 
among multiple input ports. The essential techniques under traditional traffic situations (that is, short-range dependent traffic and highly busty traffic situations (that is, long-range dependent traffic) is optical buffering have been proposed in order to reduce the PLP to an acceptable level (Danielsen et al.,1998; He et al., 2000). The effect of the packet loss is packet loss produces errors, jitter, broken up imaged.

In the network, an application generates data flows to be transported to a similar application at the other end of the network. The flows will be segmented into packets, e.g., IP packets; part of them may be further segmented into ATM cells in IP over networks. In order to be successfully arriving destination all packets composing a flow must be reconstructed into the original message at the transport layer. Later packets in a flow may be corrupted, different delays, out of order, or be lost in intermediate routers but higher layer such as transmission control protocol (TCP) has to recover these corrupted flows before passing to applications. Even though the current internet uses much electronic buffering in intermediate switching nodes to reduce packet loss, recent study by Bennett et al. (1999) focused on the internet traffic found that a considerably high number of incidences on packet re-ordering in end systems due to local parallelism existing in the nodes, where a packet in a flow can follow different paths between input and output ports.

OPSNs are packets usually routed and transmitted separately. Wavelength channel conversion allowed packets in a flow can be allocated to different paths to transmit in different wavelength channels in an OPSR. Also, the multi-path routing, which packet can be allocated to different paths to transmit in different fibers. Both cases can drive the packet out of order and as a result cause high-level applications to failure. Although low PLP could be achieve with optical buffering (Hluchyj and Karol, 1988), researchers prove it is far from being capable of achieving good performance regarding higherlayer protocols especially under the circumstances of packet overcrowding.

Therefore, researchers propose two oriented discard techniques flow discards (FD) and early flow discards (EFD) for flow routing networks which are expected to prevent the corrupted flow from overwhelming the network resources. FD rejects the entire flow while the remaining buffer capacity is not large enough to accommodate it, when EFD discards the entire flow each time the buffer occupancy exceeds some level. In addition, the OPSR the performance of the packet discards (PD) is investigated without flow control.

\section{Literature review}

It should be noted that since it would be very complicated for a high-speed router to maintain the routing status, keep the packet order, and remain the same delay, packets are usually transmitted without any control mechanism in OPSRs. Thus, this section extensively discusses on routing and switching techniques in OPSR. Moreover, special focus on corrupted containing an outof-order packet(s), variously delayed packet(s) and/or packet missing(s) are highlighted.

\section{Optical buffering}

Optical buffering can be generally classified into three categories namely input buffering; output buffering, shared/ partially shared buffering.

\section{Input buffering}

With input buffering each input channel has a buffer queue. Under the First-in-First-out (FIFO) service discipline, when two or more packets from different channels destine to the same output channel at the same time, one of them are directed to the output while the others have to remain in the queues until the output is available. Therefore, the following packets in those queues are blocked. This is called head-offline blocking, which results in a maximum throughput of 0.586 for an input-buffered router (Lamaire and Serpanos, 1994). To solve that, most input-buffered switches require input ports to bid for output ports in parallel, combined with a good arbiter (Danielsen et al., 1997). Practically, the arbiter introduces randomness that unavoidably gives rise to packet out-of-order.

\section{Output buffering}

With output buffering, each output channel has a buffer queue. Packets destine to an output will be stored in its buffer until the channel is available. Output buffering exhibits the optimal delay/throughput performance (Guillemot, 1998). It can retain the packet order under FIFO. However, assuming if the first packet of the arrive at the buffer together with packets from other input ports, they will occupy the buffer position one after another. The second packet of the flow has to stay in a buffer position, interleaved from the first packet. Therefore, they experience different delays. Additionally, low PLP can be achieved with optical buffering under the short-range dependent traffic (Danielsen et al., 1998).

\section{Shared/partially-shared buffering}

In this case, the router comprises a central buffering system or a set of shared buffers (opposite to the prime buffer of each output) to be shared among the outputs 


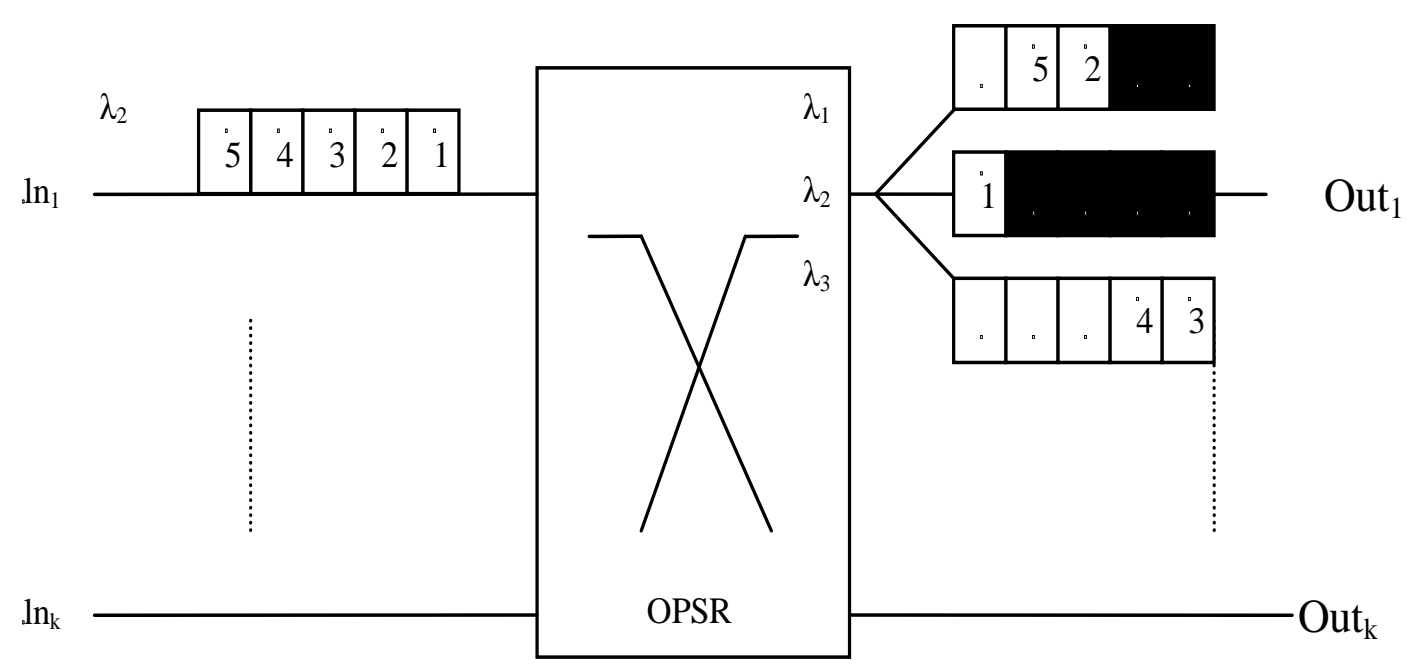

Figure 1. Deployment of wavelength conversion in the optical packet switching router.

(Bennett et al., 1999; He et al., 2000). All the packets can be stored in re-circulation delay lines of appropriately selected length in the sharing case, or only those packets that cannot be accommodated by the prime buffer will be stored in the separate buffers in the partial-sharing case. It has been shown that without wavelength converters the performance in these two cases is comparable to that in output buffering combined with wavelength conversion (Bennett et al., 1999). However, the control network of the router needs to maintain all the states of the stored packets, which requires a complex algorithm. In addition, to retain the packet order and the same delay, not only the control network's complexity is to be increased dramatically, but a random access to buffer is also needed, which is not amenable to optical implementation yet.

\section{Wavelength conversion}

Wavelength conversion has been widely studied for OPSRs. It was reported that the use of optical buffers could be reduced largely by deploying wavelength converter under both short- and long-range dependent traffic (Karol, 1993).

With wavelength conversion, concurrent wavelength channels in a fiber are available to packets destined to the same output port. Figure 1 illustrates wavelength conversion for an OPSR with three wavelength channels per fiber. A flow of five packets from $\left(\ln _{1}, \lambda_{2}\right)$ destines to $\left(\right.$ Out $_{1}, \lambda_{2}$ ). For simplicity, we assume that all the packets are of the same size, namely one time slot. Packet 1 goes into $\lambda_{2}$, which is the original path to the destination for the flow. However, in the next time slot the buffer in $\lambda_{2}$ is full, so packet 2 is randomly wavelength-converted to $\lambda_{1}$ for which the buffer is available, while packets 3 and 4 are put into $\lambda_{3}$ and packet 5 into $\lambda_{1}$ as shown in Figure 1 . This has resulted the packet loss being prevented by wavelength conversion, the sequence of the packets of the flow is broken. Hence, re-ordering is required in the end system.

\section{Flow routing}

Flow routing is used for optical IP networks. The significant different between packet switching and flow routing is that, it is based on 'flows' with fours characteristics as follow:

(i) A flow framed of packets, it dealt as one single entity for routing and switching,

(ii) The order of packets is maintained and the same delay for all the packets within the flow is guaranteed without using complicated control mechanism,

(iii) The flexibility and scalability of packet switching are retained,

(iv) It offers an application-friendly situation and increases the network performance, e.g., the good throughput (Figure 2).

\section{Model}

An analytical model claimed to be proposed for the analysis is not clearly proved with respect to how it captures the algorithms being investigated

Packet loss probability is the example of OPS network performance interest has been focused on the packet/cell level. Therefore, packet-level measurement may not be appropriate for higher-layer protocol's performance, for 


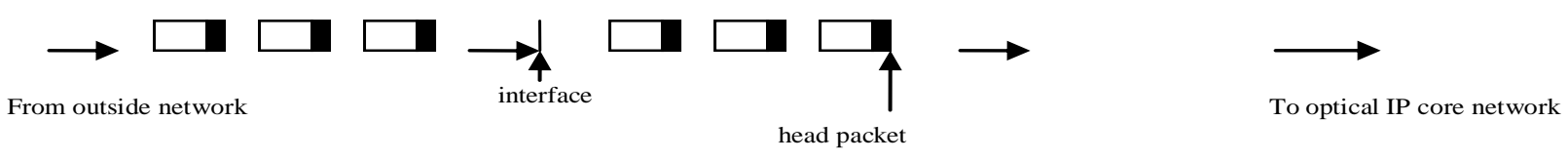

Figure 2. Flow generation in the edge router of optical flow-routing network.

\section{G vs load}

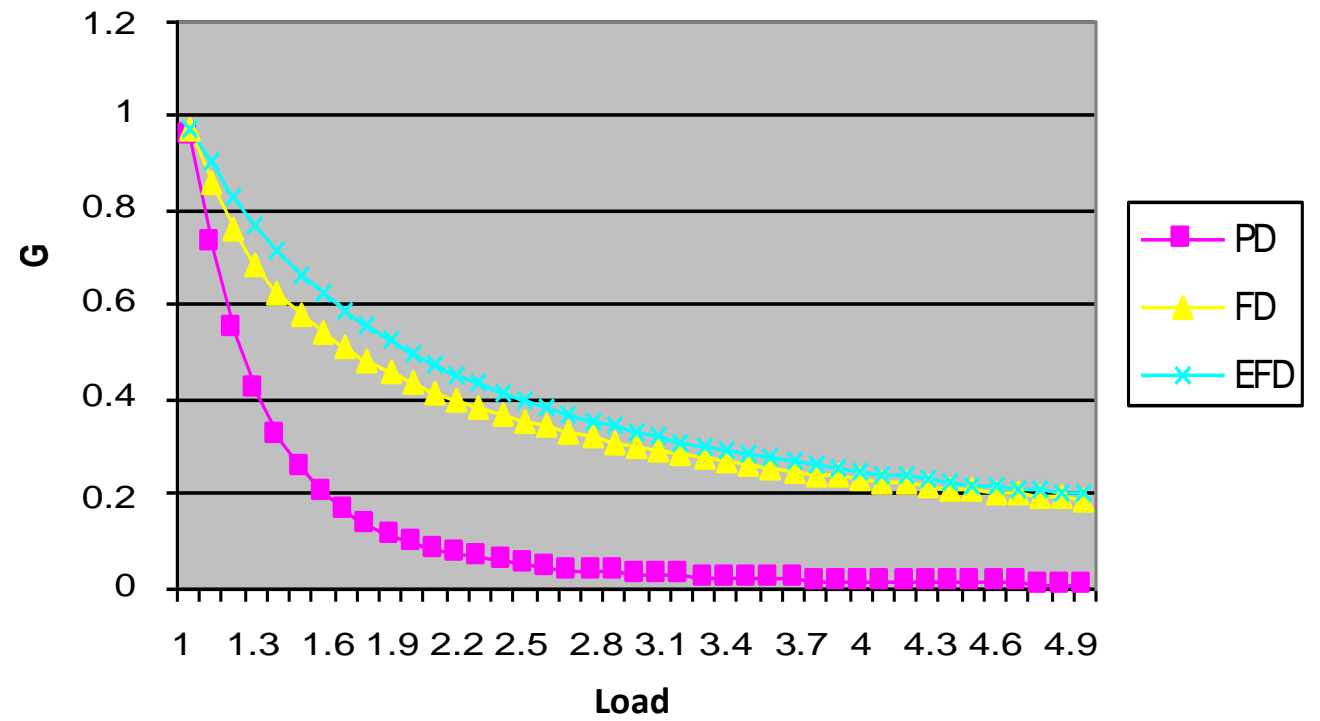

Figure 2a. Good-throughput $\mathrm{G}$ vs. load $r$ for $E F D$, FD, and PD, $\mu=1 ; B=80 ; k=50 ; L=10$ for $q=1.5$.

example, corrupted flows caused by the lost and out-oforder packets. A performance metric, good throughput (G) is acquired here. According to Lapid et al. (1998), good throughput $(G)$ is the ratio of the sum of the packets composing the flows being successfully transmitted by a router to the total packets arriving at the router's input. This metric represents the percentage of the user data that are successfully transmitted by the router. Two discarding techniques have been investigated for OFR routers, that is, FD and EFD. FD occurs due to nonenough available buffers; all of the packets composing a flow are discarded when the available buffers are less than the length of the flow upon its arrival.

$$
\operatorname{Pr}\{x=i\}=\left\{\frac{q \cdot(1-q)^{i-1}}{\sum_{j=1}^{L} q \cdot(1-q)^{j-1}}\right\} \quad \mathrm{i} \leq \mathrm{L}
$$$$
\mathrm{i}>\mathrm{L}
$$

This is able to discard the corrupted flow and save the resources for the coming traffic. With EFD, instead of discarding packets when the buffer is not enough, the router starts to discard flows when the occupancy of the buffer exceeds a threshold $k$. To compare the performance with OPS routers, a conventional packet discards (PD) technique has also been investigated. Researchers assume such a packet arrival process as a Poisson distribution with $\lambda^{\prime}$. A router model with FIFO output buffering is considered. The transmission time of a packet is exponentially distributed with mean $1 / \mu$ in time slot. Hence, in terms of packets, the queue can be viewed as an $M / M / 1 / B$ model, with arrival rate $\lambda^{\prime}$, service rate $\mu$, and buffer depth $B$ in time slot. The number of packets per flow is a shortened geometric distribution with parameter q, having probability density function of zero above $L$, where $L$ is the maximum number of packets allowed in a flow. $L$ is related to $B$ and $\mu$ as $L / \mu$ $\leq \mathrm{B}$. The load on the queue is defined as $\rho=\lambda / \mu$, where

$$
\begin{aligned}
& \lambda=\lambda^{\prime} \cdot\left[1-q \cdot \sum_{j=L+1}^{\infty} q \cdot(1-q)^{j}\right] \\
& \lambda=\lambda^{\prime} \cdot\left[1-q \cdot(1-q)^{L}\right]
\end{aligned}
$$

\section{RESULTS AND DISCUSSION}

Analytical model based on discarding technique has been used and discussed in this section. From analytical models as shown in Figure 2a the performance matrices 


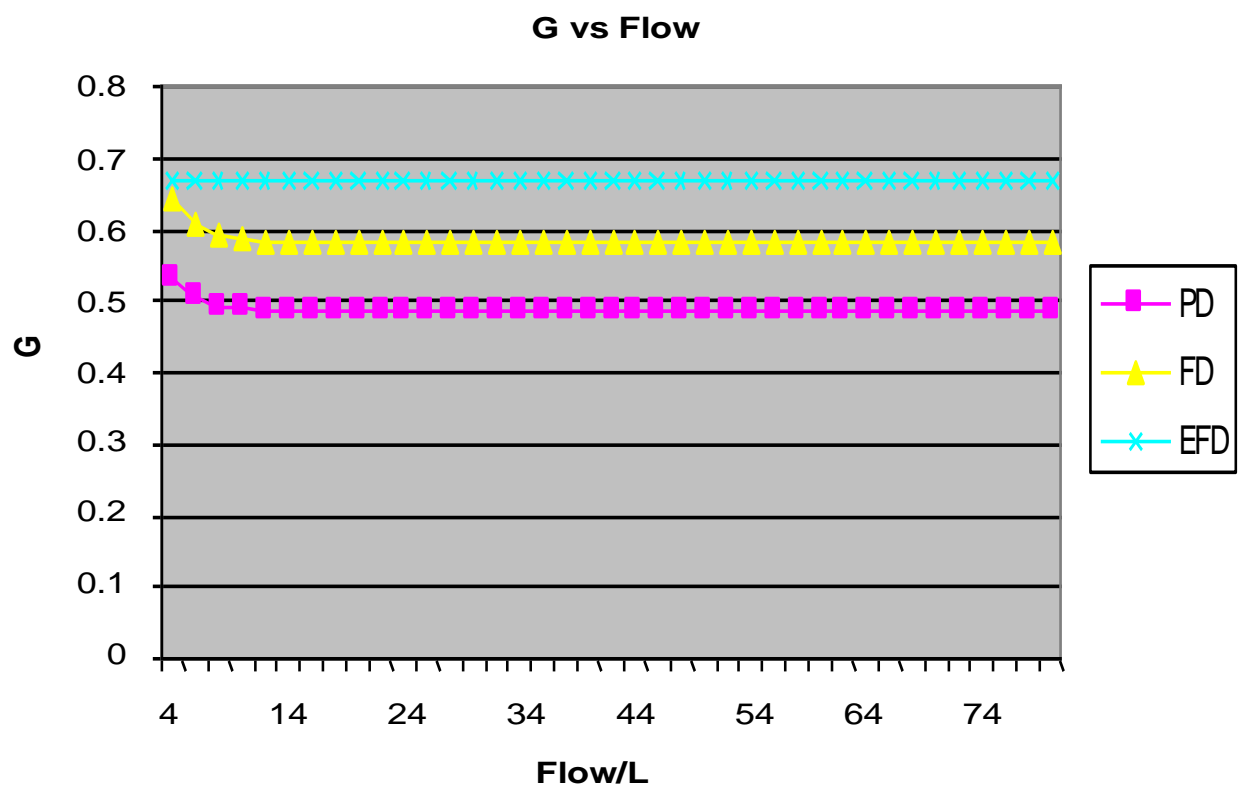

Figure 2b. Good throughput $G$ vs. flow/L for EFD, FD, and PD, $\mu=1 ; B=80 ; k=50 ; L=10$ for $q=1.5$.

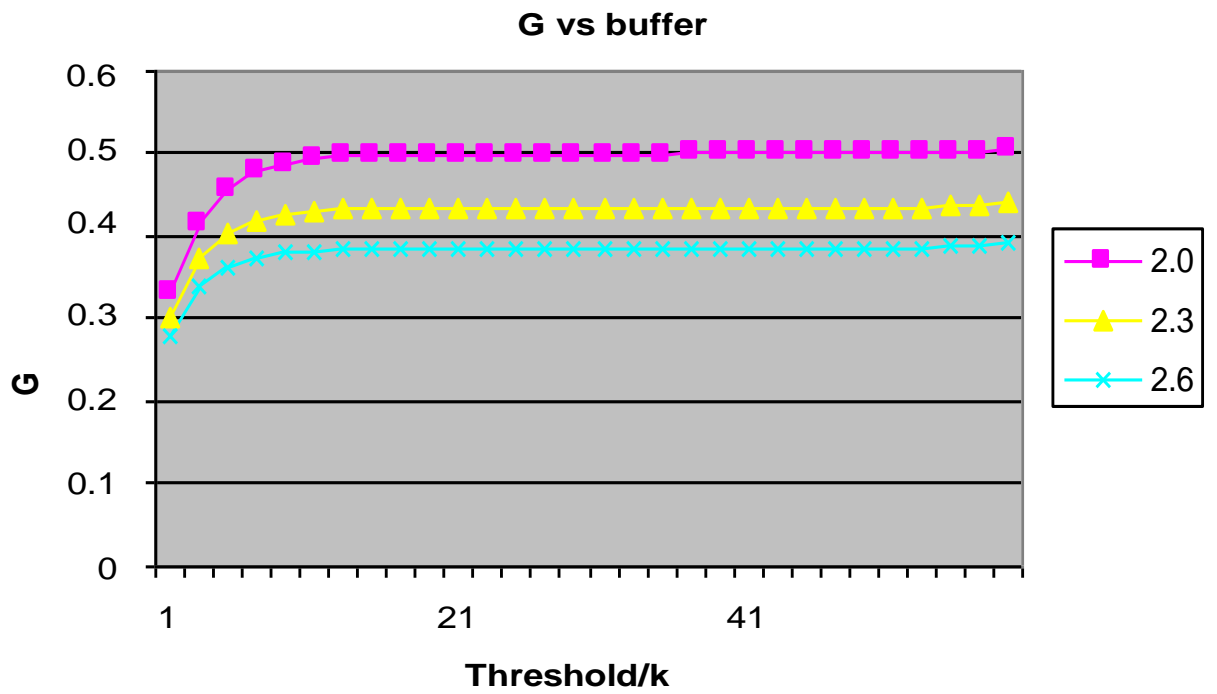

Figure 2c. Good-throughput $G$ vs. threshold/k for EFD, FD, and PD, $\mu=1 ; B=80 ; k=50$; $L=10$ for $q=1.5$.

of the goodput will affect the varieties of traffics. The factor which is consisting of PD, FD and EFD will be investigated. From the results obtained in the graph good throughput $(G)$ of the router versus load $(\rho)$ with EFD, FD and PD have been plotted based on varies loads $(\rho)$. From the graph, it is found that as the $\rho$ increases then $G$ decreases. There are three techniques that will be considered. The first case shows that G for PD declines faster than FD and EFD as shown for $r=5$ (heavy congestion) the throughputs are $20 \%$ for EFD, $19 \%$ for
FD and PD $2 \%$.

Figure $2 b$ shows the effect of the flow $(L)$ versus the good throughput $(G)$ for PD, FD and EFD. It is shown that the EFD has no effect compare to the FD and PD. Figure $2 c$ shows the effect of the threshold $(K)$ versus the good throughput (G) for EFD is plotted with under different loads. It is clearly that $G$ is insensitive to the change in a large range of $\mathrm{k}$, which means that choosing the threshold is not critical for EFD. However, with a lower threshold will also produce lower good throughput as 


\section{G vs buffer}

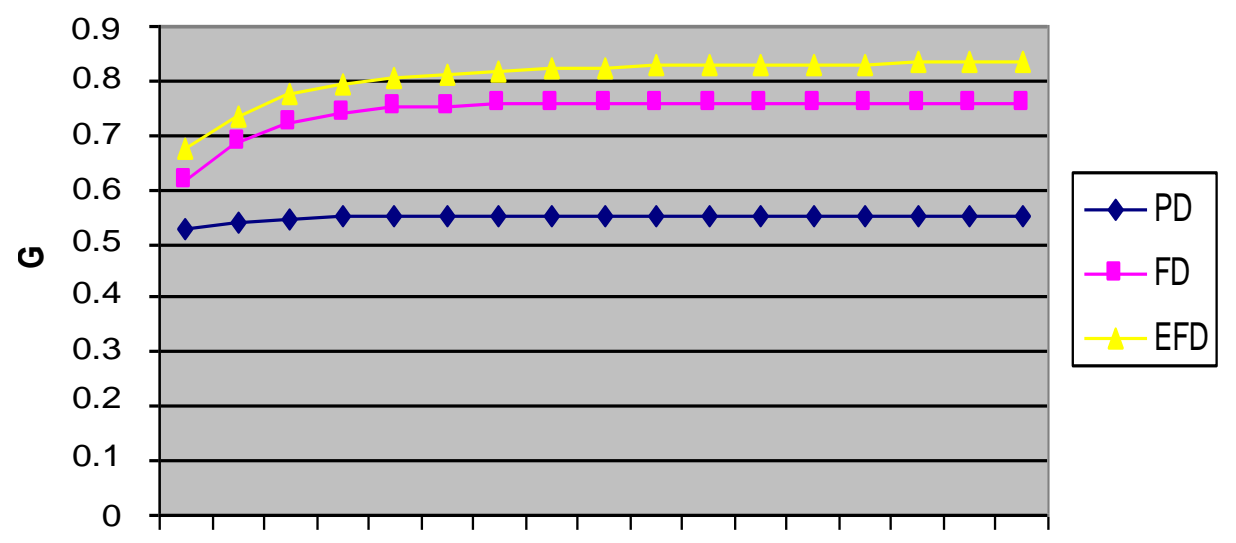

$\begin{array}{lllllllllllllllll}16 & 21 & 26 & 31 & 36 & 41 & 46 & 51 & 56 & 61 & 66 & 71 & 76 & 81 & 86 & 91 & 96\end{array}$

Figure 2d. Good-throughput G vs. Buffer/B for EFD, FD, and PD, $\mu=1 ; B=80 ; k=50 ; L=10$ for $q=1.5$.

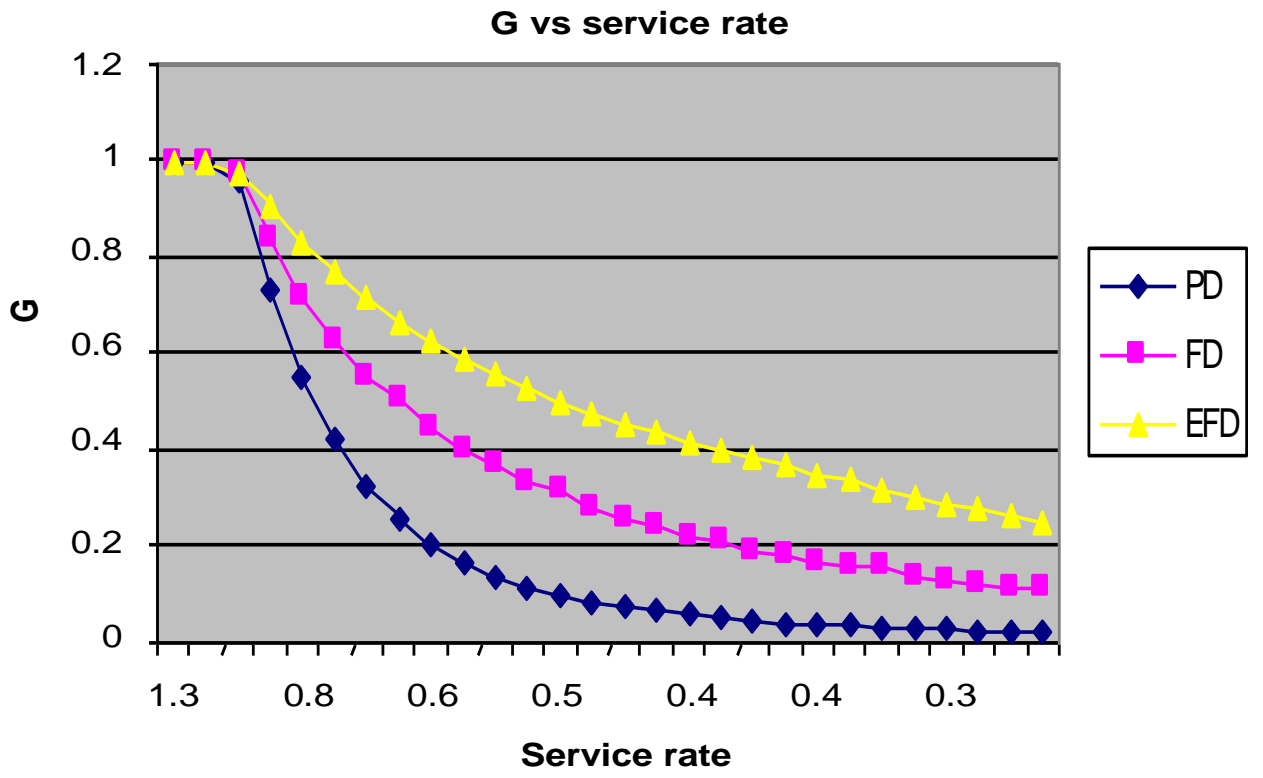

Figure 2e. Good-throughput $G$ vs. service rate/ $\mu$ for EFD, FD, and PD, $\mu=1 ; B=80 ; k=50$; $L=10$ for $q=1.5$.

well. Figure $2 d$ depicts the goodput $(G)$ versus the buffer depth (B) under a load of 1.5. The plot indicates with the increase of $B, G$ tends to increase. However, the magnitude increase of $G$ for EFD and FD are much larger than that of PD. Saturation points exist for these three cases. It can be evidenced where the saturation points appear. This result can be used to design future. Figure $2 e$ shows the effect of service rate $(\mu)$ representing the good throughput $(G)$ versus service rate $(\mu)$. From the graph it was found that as the $\mu$ increases then $G$ decreases. EFD decrease slower compares to the FD and PD.

Finally, the probability of a flow with the different number of packets being successfully transmitted by the router (Ps) is plotted in Figure $2 f$ under a load of 1.5. As shown, Ps is nearly kept at the same value for EFD. However, for PD and FD the shorter flows get the higher service possibility (high Ps), while the longer ones get the lower service possibility (low Ps). The difference between the highest and the lowest is considerably large. It is found that this difference decreases with the load decreasing. These results reveal that EFD is the fairest 


\section{Ps vs no of packet in flow}

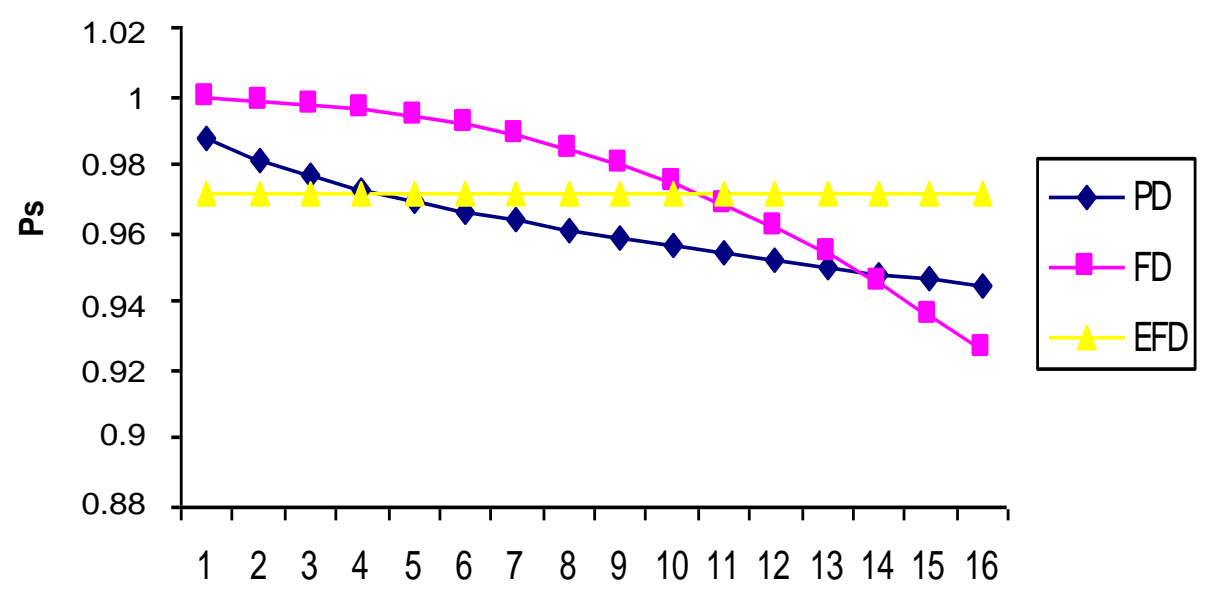

Packet flow

Figure 2f. Probability of a flow vs packet of flow/ $q$ for EFD, FD, and PD, $\mu=1 ; B=80$; $k=50$; $L=10$ for $q=1.5$.

approach among the three discarding (PD, FD, and EFD).

In conclusion, optical flow routing can be evaluated using the analytical and proposed method. Analytical method revealed that the effectiveness of EFD for good throughput compared with other conventional PD and FD. This new method is proposed suitable for the optical network due to maintaining packet order during transmission in the systems. From the results as shown in Figure $2 \mathrm{a}$ the performance good throughput of routers can be improved significantly by deploying flow routing especially under congestion periods. It can be concluded that EFD is a suitable technique for optical flow-routing networks compared to PD and FD.

\section{ACKNOWLEDGEMENTS}

Special thanks to Dr Jenny for her help and assistance throughout this study investigation. Not to forget, special thanks to my principle supervisor Prof. Dr. Kaharudin for his support, supervision and advice. It would be very difficult to finish writing up this paper without their unconditional guidance.

\section{REFERENCES}

Bennett JCR, Patridge C, Shectman N (1999). Packet reordering is not pathological network behavior. IEEE/ACM Trans. Netw. 6(7):789-798.

Carena A (1998). OPERA: An optical packet experimental routing architecture with label swapping capability. J. Lightw. Technol. 12(16):2135-2145.
Danielsen S, Mikkelsen B, Joergensen C, Durhuus T, Stubkjaer K (1997). WDM packet switch architectures and analysis of the influence of tuneable wavelength converters on the performance. J. Lightw. Technol. 5(15):219-227.

Danielsen SL, Joergensen C, Mikkelsen B, Stubkjaer KE (1998). Analysis of a WDM packet switch with improved performance under bursty traffic conditions due to tuneable wavelength converters. J. Lightw. Technol. 5(16):729-735.

Guillemot C (1998). Transparent optical packet switching: The European ACTS KEOPS project approach. J. Lightwave Technol. 12(16):2117-2134.

He JJ, Simeonidou D, Chaudhry S (2000). Contention resolution in optical packet-switching networks: Under long-range dependent traffic, Proc. of OFC'2000 (Baltimore, US, March, pp. 295-297.

Hluchyj MG, Karol MJ (1988). Queueing in high-performance packet switching. IEEE J. Selected Areas Commun. 9(6):1587-1597.

Karol MJ (1993). Shared-memory optical packet (ATM) switch, Proc. of SPIE symposium on multi-gigabit fiber communication systems, 2024:212-222.

Lamaire R, Serpanos D (1994). Two-dimensional round-robin schedulers for packet switches with multiple input queues. IEEE/ACM Trans. Netw. 5(2):471-482.

Lapid Y, Rom R, Sidi M (1998). Analysis of discarding policies in highspeed networks. IEEE J. Selected Areas Commun. 5(16):764-777.

Guoqiang H (2009). "Quality of Service Guarantee in the Edge Node of Optical Packet/Burst Switched Networks with Traffic Assembly", Optical. 2009.

Thomas SA (1996). Ipng and the TCP/IP Protocols Implementing the Next Generation Internet. New Jersey: John Wiley \& Sons Inc. 